\title{
THROMBOCYTOPENIA AND ELEVATED ALANINE AMINOTRANSFERASE LEVELS IN MALARIA PATIENTS
}

\author{
Sayed Tanveer Abbas Gilani, Amjad Khan, Tariq Ali Khan, Muhammad Farooq \\ Pak Med Level II+ Hospital XIII, UN Mission, Liberia
}

\begin{abstract}
Objective: To study thrombocytopenia and elevated ALT levels in malaria patients reporting to Pak Med Level II+ Hospital XII and XIII, UN Mission Liberia, West Africa.

Study Design: Cross sectional study.

Place and Duration of Study: Departments of Pathology and Medicine, Pak Med Level II+ Hospital XII and XIII, UN Mission Liberia, West Africa, from Feb 2015 to Dec 2016.

Methodology: In total of 100 febrile patients of both gender and all ages reported to Pak Med Level II+ Hospital XII and XIII, Liberia with clinical features of malaria and having positive malarial parasite (MP) on any of the methods of immune chromatography (ICT) or microscopic film were included. The febrile patients with typical clinical features of malaria but having negative MP both on ICT and microscopic methods were excluded. Pretreatment whole blood in EDTA was collected for testing MP on ICT and by microscopic method including MP index and platelets, while serum for ALT.

Results: Out of total 100 patients, 75 were males, 25 females with mean age of $38 \pm 5$ years. MP was found positive on MP film in $95 \%$ and on ICT in $88 \%$ cases. Thrombocytopenia was found in $69 \%$ and ALT was elevated in $60 \%$ cases. Mean and SD for platelets count was $129 \pm 72 \times 10^{9} / \mathrm{L}$ and of ALT $76 \pm 66 \mathrm{U} / \mathrm{L}$. Mean of MP index was 0.49 ranging from 0 to $3.5 \%$. Correlation of MP index with platelets and ALT was found insignificant, although in malaria patients, platelets and ALT was inversely correlated significantly with each other showing low platelets along with elevated ALT levels.

Conclusion: In malaria patients, thrombocytopenia and elevated ALT are frequent findings, which may not definitely correlate with MP index. So it is advisable to test for platelet count and ALT in all patients of malaria for early diagnosis and better management.
\end{abstract}

Keywords: Malarial parasite, MP index, Platelets, Serum ALT.

This is an Open Access article distributed under the terms of the Creative Commons Attribution License (http://creativecommons.org/licenses/by/4.0), which permits unrestricted use, distribution, and reproduction in any medium, provided the original work is properly cited.

\section{INTRODUCTION}

Malaria is a mosquito borne disease caused by plasmodium parasite. WHO report in 2015 on malaria showed 214 million malaria cases globally with 88 percent from Africa ${ }^{1}$. In west Africa, malaria leads to morbidity and mortality with $38 \%$ of outpatients and $42 \%$ of inpatient deaths 2 . In Liberia more than $85 \%$ malaria cases are due to plasmodium falciparum while only small number of cases caused by plasmodium ovale ${ }^{3}$.

Various studies revealed that hematological and biochemical abnormalities occur due to malaria causing various complications in these

Correspondence: Dr Sayed Tanveer Abbas Gilani, Consultant Chemical Pathologist, AFIC Rawalpindi Pakistan

Received: 19 Jun 2017; revised received: 26 Aug 2019; accepted: 11 Sep 2019 patients. In malaria infections, haematological changes are anemia, thrombocytopenia, and disseminated intravascular coagulation ${ }^{4,5}$. Physiochemical abnormalities due to plasmodium falciparum may vary with the level of malarial immunity, malaria endemicity, haemoglobinopathies, nutritional status and demographic factors 6,7 . The postulated mechanisms causing thrombocytopenia are coagulation disturbance, sequestration in spleen, platelet destruction by antibodies, oxidative stress and role of platelets as the cofactors, leading to severe malaria. Platelets structural and functional abnormalities have been described due to malaria and in few cases platelets can be invaded by malarial parasites ${ }^{5-7}$.

Changes in hepatocytes due to infection by the sporozoite form of the malarial parasite can 
cause the leakage of parenchymal transaminases and membranous alkaline phosphatase (ALP) from the liver. So increase in aspartate transaminase (AST), alanine aminotransferase (ALT) and ALP found in malaria patients also revealed that the serum levels of these liver enzymes increased with the increase in the density of malarial parasite. The hepatic stage of the life cycle of malarial parasite in the human is accompanied by disturbances in the hepatocyte's parenchyma and membrane, leading to leakage of liver enzymes into general circulation ${ }^{8,9}$.

Therefore, timely investigations to know the changes in haematological parameters and hepatic functions enable the clinicians to establish diagnosis and therapeutic interventions in malarial patients. Hence the aim of this study is to know the changes of platelets count and ALT in patients infected with malarial parasite in the western African region.

\section{METHODOLOGY}

This cross-sectional study was carried out at the department of Pathology and Medicine, Pak Med Level II+ Hospital XII and XIII, UN Mission Liberia, West Africa from Feb 2015 to Dec 2016 after approval of the institutional ethical review board (IERB \#5). Sample size was calculated using a standard formula of $\mathrm{WHO}$ sample size calculator. Briefly, using the prevalence of malaria as more than $88 \%$ in Africa1, precision 0.05 $(5 \%)$ and statistical power $80 \%$. In total of 100 patients of both gender and all ages reporting consecutively to Pak Med Level II+ Hospital XII and XIII, Liberia with febrile illness and diagnosed as having positive malarial parasite (MP) on any of the methods of immune chromatography (ICT) or microscopic film were included in the study. The febrile patients presenting with typical clinical features of malaria (high grade intermittent fever along with rigors and chills) but had negative MP both on ICT and microscopic film methods were excluded. Patients with history of any medical illness causing increase in liver enzymes and lowering platelets, were excluded from the study.
One hundred samples were collected consecutively from all the patients after their informed consent for the workup of malaria. History of illness, findings of the physical examination, demographic data and baseline investigations were performed at the start of the study. All the samples were collected in vacutainer tubes (BD, NJ USA). Pretreatment whole blood in tubes containing EDTA as anticoagulant was collected for testing MP by ICT [anti histidine rich proteinII (anti-HRP-II) plasmodium falciparum specific/ p.f and anti aldolase antibodies / pan line for all malarial parasite antigens] and microscopic film method including MP index. Rapid diagnostic test is a device that detects malaria antigen in a small amount of blood, usually $5-15 \mu \mathrm{L}$, by immuno-chromatographic assay with monoclonal antibodies directed against the target parasite antigen and impregnated on a test strip. The result, usually a colored test line, is obtained in 520 min. ICT methods have sensitivity of $87 \%$ and specificity of $97 \%$. In microscopic film method including MP index, Leishman stain was used and all the slides were seen and verified by pathologist. Microscopic film methods have sensitivity of $95 \%$ and specificity of $99 \%$. While platelet count was measured using automated (SYSMEX. KX-21n) hematology analyzer.

Blood samples were allowed to clot and centrifuged for $10 \mathrm{~min}$ at $1,000 \mathrm{~g}$ for analysis of serum ALT by photometric kinetic method on Microlab 300. The serum was separated and then stored at $-20^{\circ} \mathrm{C}$ until analyzed. Platelet count of less than $150 \times 10^{9} / \mathrm{L}$ was taken as thrombocytopenia and serum ALT more than $42 \mathrm{U} / \mathrm{L}$ was considered to be elevated.

Statistical analysis of all data was entered in the statistical package for social sciences version 17 (SPSS Inc, Chicago, IL, USA). Mean and SD were calculated for the quantitative variables like age, platelets and ALT levels. Mean and range was calculated for MP index. Frequencies and percentages were calculated for the qualitative variables like gender and MP by ICT and microscopic method. Pearson correlation and linear regression curve estimation was analyzed 
between MP index and platelets, ALT and also between platelets and ALT (A $p$-value of $<0.05$ in two tailed was considered significant).

\section{RESULTS}

Out of total 100 patients, 75 (75\%) were males, $25(25 \%)$ females with mean age of 38 , ranging from 22 to 50 years in both gender. MP was found positive on microscopy in 95 (95\%) cases while ICT malaria positive in 88 (88\%). Thrombocytopenia was found in $69(69 \%)$ and ALT was elevated in $60(60 \%)$ cases. Mean and SD of platelets was $129 \pm 72 \times 10^{9} / \mathrm{L}$ and of ALT $76 \pm 66 \mathrm{U} / \mathrm{L}$. Mean MP index was $0.49 \%$ ranging from 0 to $3.5 \%$ (table).

Table: Baseline characteristics of malarial patients (n=100).

\begin{tabular}{l|c}
\hline Parameter Qualitative Variables & n (\%) \\
\hline Male & $75(75 \%)$ \\
\hline Female & $25(25 \%)$ \\
\hline $\begin{array}{l}\text { MP positive on MP film by } \\
\text { microscopy }\end{array}$ & $95(95 \%)$ \\
\hline MP positive on ICT & $88(88 \%)$ \\
\hline Type of MP (Falciparum) & $100(100 \%)$ \\
\hline Thrombocytopenia & $69(69 \%)$ \\
\hline Increased ALT levels & $60(60 \%)$ \\
\hline Quantitative Variables & Mean \pm SD \\
\hline Age (years) & $38 \pm 5$ \\
\hline Platelets count (x 10\% $/ \mathrm{L})$ & $129+72$ \\
\hline Serum ALT (U/L) & $76 \pm 66$ \\
\hline & Mean (Range) \\
\hline MP index $\%$ & $0.49(0-3.5)$ \\
\hline
\end{tabular}

Correlation of MP index with platelets and ALT was found insignificant showing $p$-value more than 0.05 and r-values were 0.025 and 0.001 , respectively. Although in malaria patients platelets and ALT was significantly correlated inversely with each other showing low platelets and increased ALT levels ( $p$-value less than 0.05 and R value 0.083 ) (fig-1, 2 \& 3).

\section{DISCUSSION}

Malaria usually affects all the blood components and is known as a haematological infectious disease ${ }^{10}$. Anemia and thrombocytopenia are most frequent haematological complications while elevated transaminases are common bio- chemical manifestations associated with malaria. Thrombocytopenia is defined as the platelet

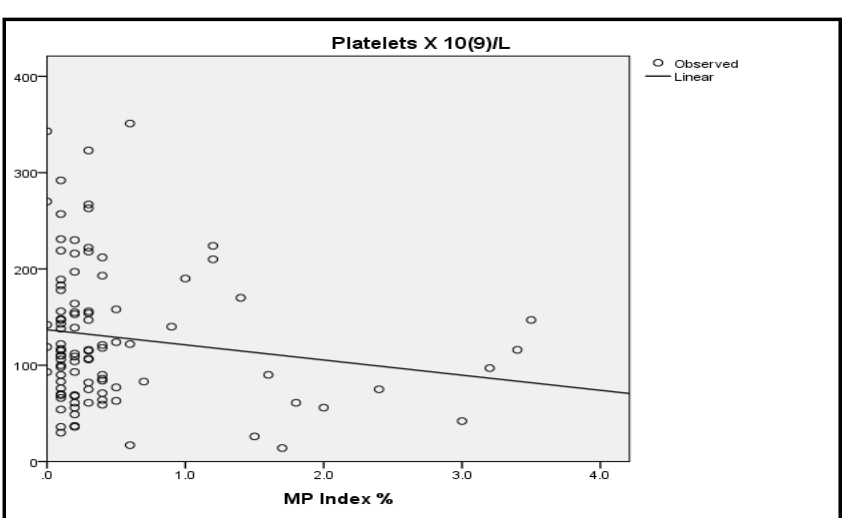

Figure-1: Insignificant inverse correlation of MP index and platelets in malarial patients showing $p$ value 0.118 and $r$-value is $0.025(n=100)$.

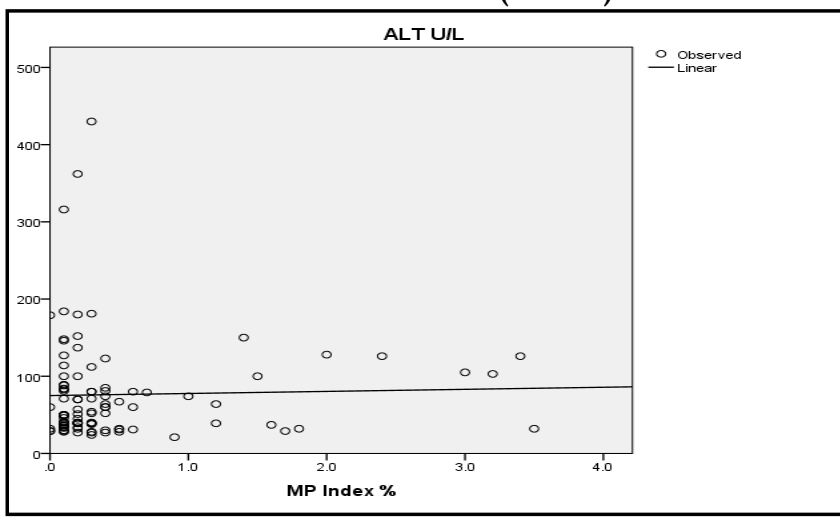

Figure-2: Insignificant correlation of MP index and ALT in malarial patients showing $p$-value 0.77 and $r$-value is $0.001(n=100)$.

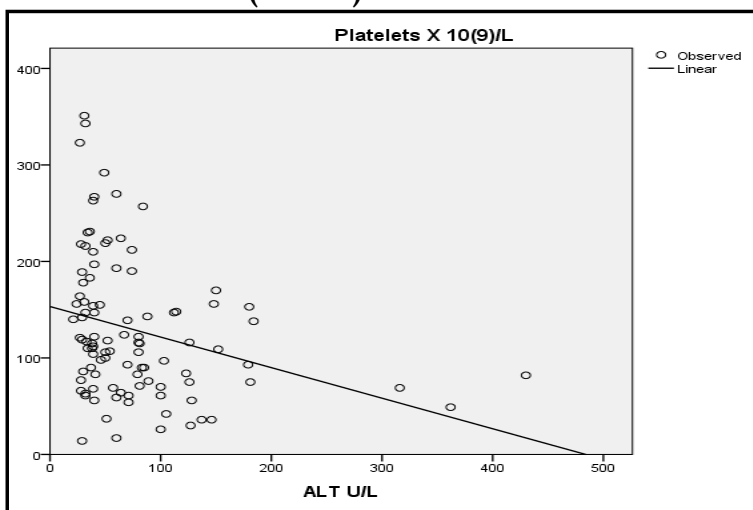

Figure-3: Significant inverse correlation of elevated ALT and low platelets in malarial patients showing $p$-value 0.004 and $r$-value is $0.083(\mathrm{n}=100)$.

counts below $150 \times 10^{9} / \mathrm{L}$ and is frequent finding among malaria patients ${ }^{11}$. Malaria leads to the structural and functional abnormalities in the 
platelets. Thrombocytopenia in malaria patients may be due to multiple factors which include increased platelet destruction, coagulation disturbances, sequestration in spleen, oxidative stress, as well as invasion of the platelets by malarial parasite $^{5-7}$.

In our study, thrombocytopenia was detected in $69 \%$ patients of plasmodium falciparum malaria. Several other studies also recorded similar frequencies of thrombo-cytopenia. In a study by Gupta et al in 2013 thrombocytopenia was found in $77 \%$ of patients with falciparum malaria while in $67 \%$ of cases with plasmodium vivax infection ${ }^{12}$. Another study by Colonel et al. showed thrombocytopenia in $72 \%$ of patients having malaria infection ${ }^{13}$. While studies from Saudi Arabia and Qatar by Bashawari et al in $2001^{14}$ and Joseph et al in $2011^{15}$ recorded thrombocytopenia in $53-56 \%$ cases of acute malaria. In literature review, platelet counts below $150 \times 10^{9} /$ L found in $24-94 \%$ patients with acute malaria ${ }^{16}$. These variations in values are due to the type of malarial infection by different parasite species with more severe thrombocytopenia caused by plasmodium falciparum and mixed infections. Drug resistance, geographical area and endemicity, may also cause variations as the drug resistant parasites prolong their stay in the hosts and cause damage which results in thrombocytopenia. Reduced platelets is more common than anemia in malarial infection. In our study, thrombocytopenia was found in malaria patients revealing statistically insignificant correlation between density of malarial parasite (MP index) and low platelets. Although it is noted by various studies that increased load of plasmodium falciparum parasite leads to decreased platelets count ${ }^{17}$.

The results reported in our study showed significant increase in ALT (60\%), among patients with plasmodium falciparum malaria. Our study results are as similar as the study of Sudha et al in 2014, which revealed increased serum liver enzymes (ALT 50\%, AST 53\%, and ALP 11.7\%), indicating liver damage in malaria patients ${ }^{18}$. Elevated serum ALT in malaria patients were also found in several other studies revealing $37 \%$ to $60 \% 19-21$.
Elevated liver enzymes in malaria patients could be the result of leakage of the enzymes from the damaged liver cells during the hepatic stage of the life cycle of malarial parasite. Enemchukwu et al in 2014 suggested that typhoid, malaria and typhoid-malaria combined-infections can increase AST, ALT and ALP serum levels and result in liver damage if not treated properly ${ }^{22}$.

Several studies have concluded that increase in the liver enzymes in malaria patients have positive correlation with the density of malarial parasite in the blood and severity of the disease $^{17,23,24}$. Various studies have also shown that correlation is found between parasite density and malarial infections severity 25 . In our study, the liver enzyme levels increased with increase in the severity of malaria but MP index was not as significantly correlated with ALT levels as described by studies in literature. Although our study showed that significant correlation exists between low platelets and elevated ALT levels in patients of falciparum malaria. In malaria patients, MP index is usually high at presentation but elevation of liver enzymes is late manifestation of the malarial infection and individual variations also exist. In the same way hematological manifestations, including thromb-ocytopenia may vary significantly among individuals. So it is advisable to test for MP index, platelet count and ALT in all patients of malaria to cater for individual variations and better management.

\section{LIMITATION OF STUDY}

As ALT rises late during course of the malarial infection so studies may also be performed to see correlation of MP index with ALT and platelets initially and after 4 to 5 days of presentation. Ultrasonography findings of liver and spleen may also be correlated with MP index, ALT and platelet count of plasmodium falciparum positive individuals.

\section{CONCLUSION}

The presence of the thrombocytopenia and elevated liver enzymes raises the suspicion of malaria in appropriate clinical setting. These hematological and biochemical changes should 
alert the clinician to the possibility of severe malaria and need for prompt indoor management to prevent multisystem complications due to hyperparasitemia. The study findings also demonstrate that when evaluating a febrile illness in malaria endemic zones, clinical presentation may be varied and clinician should be vigilant not to miss this important treatable condition.

\section{CONFLICT OF INTEREST}

There is no conflict of interest to be declared by any author.

\section{REFERENCES}

1. WHO. World malaria report: 2015. Available at www.who.int/ malaria

2. Liberia health facility survey. Monrovia, Government of Liberia, Ministry of Health and Social Welfare, 2009.

3. Susi B, Whitman T, Blazes DL, Burgess TH, Martin GJ, Freilich D. Rapid diagnostic test for Plasmodium Falciparum in 32 Marines medically evacuated from Liberia with a febrile illness. Ann Int Med 2005; 142: 476-77.

4. Maina RN, Walsh D, Gaddy C, Hongo G, Waitumbi J, Otieno L, et al. Impact of Plasmodium falciparum infection on haematological parameters in children living in Western Kenya. Malaria J 2010; 9(3): 4-9.

5. Chandra S, Chandra H. Role of haematological parameters as an indicator of acute malarial infection in Uttarakh and state of India. Mediterranean J Hematol Infect Dis 2013; 5(1): e2013009.

6. Price RN, Simpson JA, Nosten F, Luxemburger C, Hkirjaroen L, ter Kuile F, et al. Factors contributing to anemia after uncomplicated falciparum malaria. Am J Trop Med Hyg 2001; 65(5): 614-22.

7. Erhart LM, Yingyuen K, Chuanak N, Buathong N, Laoboonchai A, Miller RS, et al. Hematologic and clinical indices of malaria in a semi-immune population of Western Thailand. Am J Trop Med Hyg 2004; 70(1): 8-14.

8. Kochar DK, Singh P, Agarwal P, Kochar SK, Pokharna R, Sareen PK. Malarial hepatitis. J Assoc Physicians India 2003; 51: 1069-72.

9. Devarbhavi H, Alvares JF, Kumar KS. Severe falciparum malaria simulating fulminant hepatic failure. Mayo Clin Proc 2005; 80(3): 355-58.

10. George OI, Ewelike-Ezeani CS. Haematological changes in children with malaria infection in Nigeria. Int. J Med Med Sci 2011;
2(4): 768-71.

11. Lathia TB, Joshi R. Can hematological parameters discriminate malaria from nonmalarious acute febrile illness in the tropics? Indian J Med Sci 2004; 58(6): 239-44.

12. Gupta NK, Bansal SB, Jain UC, Sahare K. Study of thrombocytopenia in patients of malaria. Trop Parasitol 2013; 3(1): 58-61.

13. Colonel KM, Bhika RD, Khalid S, Khalique-ur-Rehman S, Syes ZA. Severe thrombocytopenia and prolonged bleeding time in patients with malaria (a clinical study of 162 malaria cases). World Appl Sci J 2010; 9(1): 484-88.

14. Bashawri LA, Mandil AA, Bahnassy AA, Ahmed MA, AlShamsi MA, Bukhari HA. Epidemiological profile of malaria in a university hospital in eastern region of Saudi Arabia. Saudi Med J 2001; 22(1): 133-38.

15. Joseph V, Verma M, Vidhyasagar S, Mathew A. Comparision of clinical profile and complications of mixed malarial infections of P. falciparum and P. vivax versus P. falciparum Monoinfection. Sultan Qaboos Uni Med J 2011; 11: 377-82.

16. Malik AM, Zaffar N, Ali N, Malik AM, Khan R. Haematological findings and endemicity of malaria in Gadap region. J Coll Physicians Surg Pak 2010; 20(2): 112-16.

17. Phillips A, Bassett P, Zeki S, Newman S, Pasvol G. Risk factors for severe disease in adults with falciparum malaria. Clin Infect Dis 2009; 48(7): 871-88.

18. Sudha Jha, Shrestha S, Sheetal G, Gole and Deep G. Assessment of serum bilirubin and hepatic enzymes in malaria patients. Int $\mathrm{J}$ Biomed Adv Res 2014; 5(3): 160-62.

19. Ignatius CM, Emeka EN, Blessing NE. Effect of malaria parasitaemia on liver enzyme tests. Intl J Trop Med 2008; 3(3): 49-52.

20. Onyesom I, Onyemakonor N. Levels of parasitaemia and changes in some liver enzymes among malarial infected patients in Edo-Delta region of Nigeria. Curr Res J Biol Sci 2011; 3(2): 78-81.

21. Abro AH, Ustadi AM, Abro HA, Abdou AS, Younis NJ, Akaila SI. Jaundice with hepatic dysfunction in P. falciparum malaria. J Coll Physicians Surg Pak 2009; 19(6): 363-66.

22. Enemchukwu BN, Ibe CC, Udedi SC, Iroha A, Ubaoji KI. Liver function assessment in malaria, typhoid and malaria-typhoid coinfection in Aba, Abia State, Nigeria. Pak J Biol Sci 2014; 17(6): 860-63.

23. Bhalla A, Suri V, Singh V. Malarial hepatopathy. J Postgrad Med 2006; 52(4): 315-20.

24. Salahy MA, Shnawa B, Abed G, Mandour A, Al-Ezzi A. Parasitaemia and its relation to hematological parameters and liver function among patients malaria in Abs, Hajjah, Northwest Yemen. Interdiscip Perspect Infect Dis 2016; 5954394: 1-5.

25. Tangpukdee N, Krudsood S, Kano S, Wilairatana P. Falciparum malaria parasitemia index for predicting severe malaria. Int J Lab Hematol 2012; 34(3): 320-27. 